Tecnología e Innovación

Tecnologia e Inovação

Tecnology and Innovation

\title{
ANÁLISIS COMPARATIVO DEL DISEÑO DE LA GEOMETRÍA ALAR PARA UNA GÓNDOLA AERODINÁMICA DE UN GLOBO SONDA RECUPERABLE Y REUTILIZABLE ${ }^{1}$
}

ANÁLISE COMPARATIVA DO DESENHO DA GEOMETRIA DE ASA PARA UMA GÔNDOLA AERODINÂMICA DE UM BALÃO SONDA RECUPERÁVEL E REUTILIZÁVEL ${ }^{2}$

Comparative Analysis of the Wing Geometry Design for an Aerodynamic Gondola of a Recoverable and Reusable Probe Balloon ${ }^{3}$

David Rubio Forero 4

Fundación Universitaria Los Libertadores

\section{CIENCIA Y PODER AÉREO}

ISSN 1909-7050 / E- ISSN 2389-2468 / Volumen 10 / Enero-Diciembre de 2015/ Colombia/ Pp. 1-260 Recibido: 17/01/2015

Aprobado evaluador interno: 23/10/2015

Aprobado evaluador externo: 27/11/2015

Doi: http://dx.doi.org/10.18667/cienciaypoderaereo.426

CIENCIA Y PODER AÉREO | Revista Científica de la Escuela de Postgrados de la Fuerza Aérea Colombiana | 169 
CIENCIA Y PODER AÉREO | Revista Científica de la Escuela de Postgrados de la Fuerza Aérea Colombiana |Vol. 10 | Enero - Diciembre de 2015

Para citar este artículo:

Forero, D. (2015). Análisis comparativo del diseño de la geometría alar para una góndola aerodinámica de un globo sonda recuperable y reutilizable. Ciencia y Poder Aéreo, 10 (1), 169-178. Doi: http://dx.doi.org/10.18667/ cienciaypoderaereo.426

\footnotetext{
'Artículo científico original, derivado del proyecto titulado: implementación del prototipo de un globo sonda recuperable y reutilizable para toma de datos atmosféricos. Adscrito al grupo de investigación en Ciencia Aeroespaciales-GICA. Fecha de inicio: abril de 2012. Fecha de terminación: diciembre de 2015. Financiado por la Fundación Universitaria Los Libertadores. Bogotá, Colombia.

${ }^{2}$ Artigo científico original, derivado do projeto titulado: Implementação de um protótipo dum balão sonda recuperável e reutilizável para a tomada de dados atmosféricos. Ligado ao grupo de pesquisa em Ciências Aeroespaciais -GICA. Data de início: Abril 2012 data de Conclusão: Dezembro de 2015. Financiado pela Fundação Universitária Los Libertadores. Bogotá Colômbia

${ }^{3}$ Original scientific paper, derived from the project entitled: Implementation of a prototype probe balloon recoverable and reusable for making atmospheric data. Attached to the research group in Aerospace Science-GICA. Start date: April 2012. Completion date: December 2015. Funded by the University Foundation Los Libertadores. Bogota, Colombia.

${ }^{4}$ Especialista en Gerencia de Proyectos de la Universidad El Bosque. Ingeniero Aeronáutico - Fundación Universitaria Los Libertadores, IEA 509 - M.P. CN27047060. Investigador Grupo de Investigación en Ciencia Aeroespaciales-GICA. Fundación Universitaria Los Libertadores. Correo electrónico: drubioforero@ gmail.com
}

Resumen: el presente artículo plantea dos diseños conceptuales de alas que mantienen el área alar, la razón de aspecto, la envergadura y los perfiles aerodinámicos iguales, mediante la variación de la forma alary cambiando la longitud de la cuerda en la raíz y la punta de cada modelo, con el fin de determinar el diseño con más eficiencia de la geometría alar de una góndola aerodinámica que pueda ser implementada en una radiosonda recuperable y reutilizable. Se analiza el coeficiente de sustentación y arrastre en función del ángulo de ataque, como parámetro comparativo, lo que permite evidenciar una mejora en la sustentación cuando la cuerda de la punta tiene más longitud.

Palabras clave: ala volante; cuerda; eficiencia; góndola aerodinámica.

Resumo: este artigo apresenta dois modelos conceituais de asas que mantêm a área da asa, a relação de aspecto, a envergadura e os perfis aerodinâmicos iguais, através da variação da forma da asa e alterando o comprimento da corda na raiz e na ponta de cada modelo a fim de determinar o desenho com maior eficiência da geometria de asa duma nacela aerodinâmica que possa ser implementada numa radiossonda recuperável e reutilizável. Analisa-se o coeficiente de sustentação e arrasto dependendo do ângulo de ataque como um parâmetro comparativo, o que permite evidenciar uma melhoria na elevação quando a corda da ponta tem maior comprimento.

Palavras-chave: asa voadora; corda; eficiência; nacela aerodinâmica.

Abstract: The present paper describes two conceptual wing designs that maintain equals the wing area, the aspect ratio, the wing span and the aerodynamic profiles, varying wing shape by changing the length of the root and tip chord of each model, in order to determinate the most efficient wing geometry design of an aerodynamic nacelle than could be implemented in a recoverable and reusable radio probe. The lift and drag coefficients are analyzed in function of the angle of attack, as a comparative parameter, finding an improvement on the lift when the tip chord has more length.

Key Words: Aerodynamic Gondola; Flying Wing; Efficiency; Chord. 


\section{Introducción}

El radio sondeo a nivel mundial busca conocer la estructura del estado de la atmosfera que es de fundamental importancia para interpretar las variaciones en el estado del tiempo, es por esto, que la universidad Los Libertadores adelanta un proyecto de un globo sonda recuperable pues tras el lanzamiento tradicional de dicha sonda debe esperar que el globo por diferenciales de presión sea obligado a expandirse hasta que se estalle, el globo es llevado sin dirección fija según la deriva del viento circundante mientras transmite los datos atmosféricos a una estación terrestre, la sonda debe alcanzar altitudes significativas y finalmente en su retorno la fuerza de la gravedad y los diversos vientos hacen que la sonda sea irrecuperable debido a las grandes distancias a las puede llegar en relación al punto de lanzamiento.

Lo que se pretende con el desarrollo del proyecto globo sonda es poder establecer un procedimiento rápido y frecuente de lanzamientos de globos sonda que permitan a la sociedad colombiana mejorar los estudios de la atmosfera y poder recuperar la sonda, para ello, se plantea desarrollar una góndola con forma aerodinámica de ala volante (Flying Wing), siendo esta la configuración potencialmente más eficiente de una aeronave desde el punto de vista aerodinámico y de peso estructural capaz de operar en regímenes de altas velocidades durante el descenso, que ofrece control efectivo de vuelo durante el segmento de planeo desde el momento de la liberación y luego poder ser propulsado en la altura comprendida entre los 4600 metros a cero metros, es una unidad compacta y modular, que proporcione las características aerodinámicas apropiadas para su tamaño como RE, $\mathrm{CL}$ y L/D (Stinton,1998). El diseño a desarrollar busca la configuración de planta alar más eficiente la cual debe tener la mejor senda de planeo posible para la aeronave SkyUp que albergará los sensores electrónicos para la toma de datos atmosféricos e imágenes de la curvatura terrestres desde el territorio colombiano como se muestra en la Figura 1.

\section{Criterios iniciales de diseño}

Los parámetros de diseño preliminares que se generaron para la estructura y aerodinámica de la góndola que se establecen para la determinación del diseño de la plataforma alar con área alar de 0,35 metros cuadrados, peso máximo al despegue 2200 gramos, velocidad de pérdida de 39 $\mathrm{Km} / \mathrm{h}$, carga paga de 1080 gramos. Estos valores fueron calculados para el performance anteriormente, otro de los datos establecidos por análisis fue determinar para el ala un perfil MH60 que brinda el coeficiente de momento más cercano a cero, lo ideal para este tipo de plataforma alar. Además, el valor de la relación porcentual entre la cuerda y

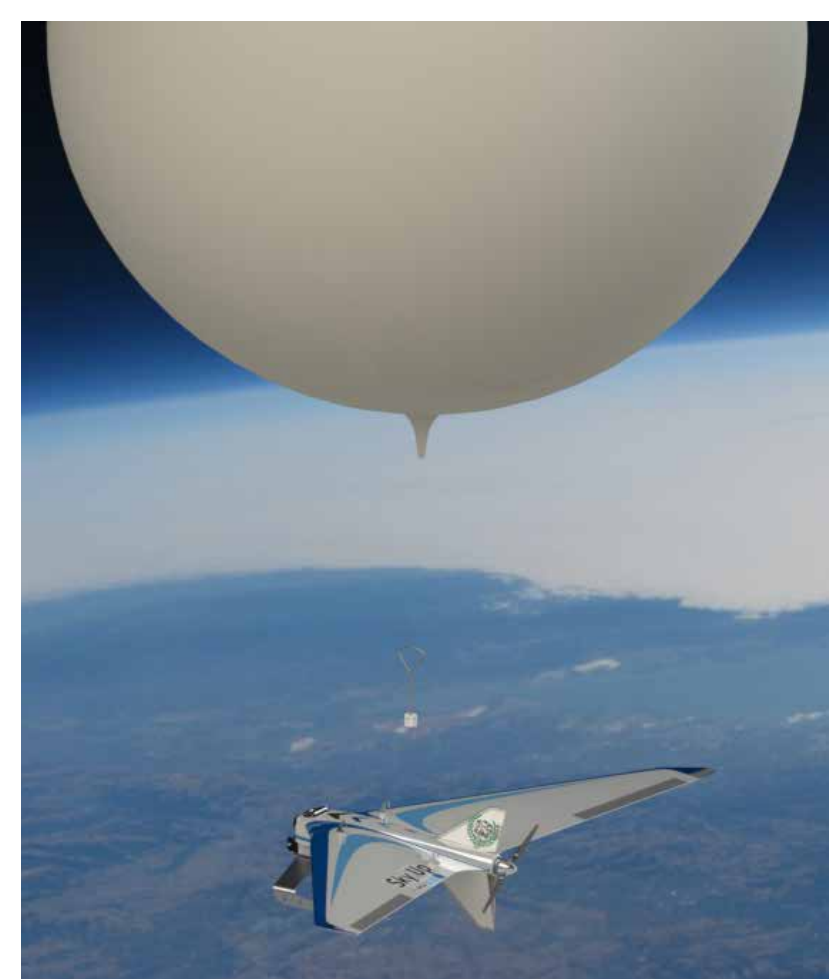

Figura 1. Imagen simulando un radiosondeo por encima de los 25.000 metros de altura. Fuente: elaboración del autor.

el espesor del perfil es adecuada para albergar una estructura interna y por ende más liviana, este perfil será empleado en la mayor parte de la extensión o envergadura de las alas, para la punta se empleará el perfil HS522 que también fue desarrollado para alas volantes y deltas con un alto ángulo de aflechamiento y lo más importante para números RE por debajo de 100000.

Para buscar la mejor senda de planeo se quiere establecer la geometría del ala ideal para el diseño estructural y aerodinámico necesario para su lanzamiento y retorno, por tal motivo se quiere analizar dos diseños de geometría alar denominados SU-1 y SU-2 partiendo de los parámetros esenciales que se han determinado para el diseño de la góndola, pero antes determinamos una de Razón de Aspecto (AR) para ambas geometrías de 6,5.

Por la experiencia que se tiene y los estudios realizados entre mayor sea el AR aumenta la sustentación, lo que mejora la senda de planeo como se muestra en la Figura 3, siendo este valor muy importante y necesario para nuestra misión, pero no hay que olvidar que a mayores AR la envergadura debe aumentar debido a que este factor depende directamente de la relación cuerda-envergadura, lo que podría complicar la construcción del prototipo si se quiere aumentar la longitud de las alas lo que haría la construcción más tediosa porque al aumentar la envergadura debe disminuir la cuerda al igual que el espesor del perfil. Lo 


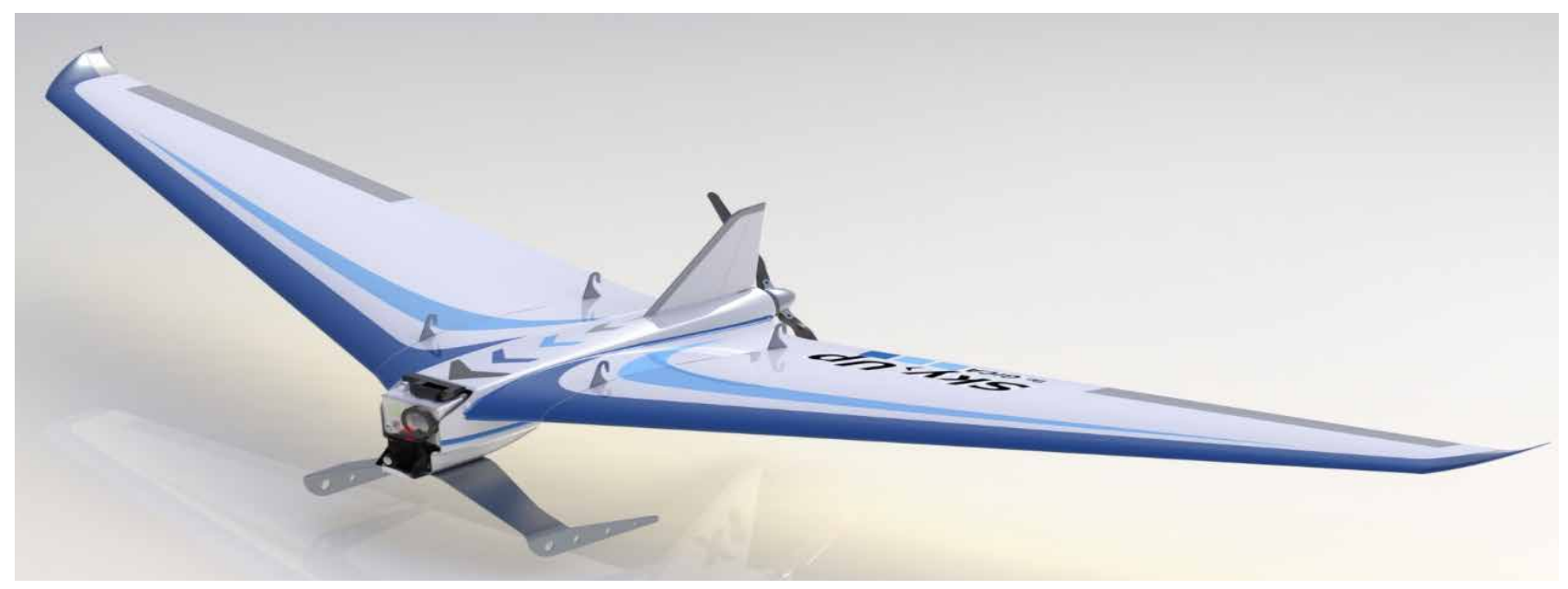

Figura 2. Configuración preliminar del diseño de la góndola aerodinámica recuperable. Fuente: elaboración del autor.

anterior, podría generar una estructura alar muy delgada y pesada, es por este motivo, que se toma un valor intermedio ente 6 y 7 ideal para construcción de prototipos.

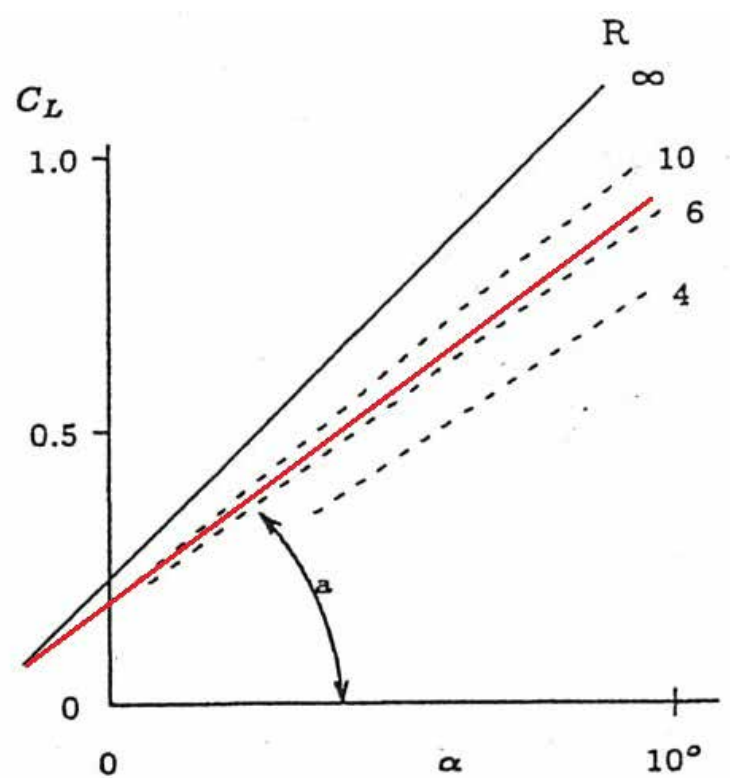

Figura 3. Efecto del Radio de Aspecto en relación con el ángulo de ataque. Fuente: elaboración del autor.

\section{Método}

Teniendo en cuenta los criterios iniciales de diseño se pretende analizar dos casos para dos modelos diferentes en los que se varía la geometría alar, el primer caso de análisis determinara el Coeficiente de Sustentación (CL) y la relación Sustentación- Arrastre (L/D) estableciendo la diferencia existente entre los dos modelos desarrollados en los cuales de vario la Razón de Taperado (TR) y el ángulo de aflechamiento; el segundo caso analiza la diferencia aerodinámica de las cuerdas principales a lo largo de la envergadura de cada modelo (cuerda en la raíz, cuerda media aerodinámica y cuerda en la punta),el número Reynolds para cada cuerda y determinar los diferentes comportamientos aerodinámicos a diferentes ángulos de ataque. Con estos valores se pretende determinar cuál puede ser el mejor modelo de geometría alar para la góndola mediante un análisis comparativo teórico de cada modelo como se muestra en las figuras 4 y 5 omitiendo por ahora el fuselaje, tren de aterrizaje y superficies de control de vuelo debido a que estos son iguales para ambos modelos.

Tabla 1. Cuadro comparativo de las geometrías alares

\begin{tabular}{ccc}
\hline Descripción & SU-1 & SU-2 \\
\hline AR & 6,5 & 6,5 \\
\hline Envergadura & $1,54 \mathrm{mts}$ & $1,54 \mathrm{mts}$ \\
\hline Área & $0.36 \mathrm{~m}^{2}$ & $0.36 \mathrm{~m}^{2}$ \\
\hline TR & 0,26 & 0,36 \\
\hline$\Lambda$ & $24^{\circ}$ & $22^{\circ}$ \\
\hline$M A C$ & $25.4 \mathrm{~cm}$ & $24.8 \mathrm{~cm}$ \\
\hline$C_{\text {raiz }}$ & $37 \mathrm{~cm}$ & $35 \mathrm{~cm}$ \\
\hline$C_{\text {punta }}$ & $9,5 \mathrm{~cm}$ & $12,4 \mathrm{~cm}$ \\
\hline
\end{tabular}

Fuente: elaboración del autor.

\section{Resultados y análisis}

Los análisis a desarrollar se basan en determinar en primera instancia el Coeficiente de Sustentación (CL) más efectivo para cada modelo y la relación que existe entre la Sustentación y el Arrastre (L/D) resultados cruciales para determinar la geometría alar en el modelo Sonda recuperable y reutilizable. 


\section{Sky, Up/SU-2}
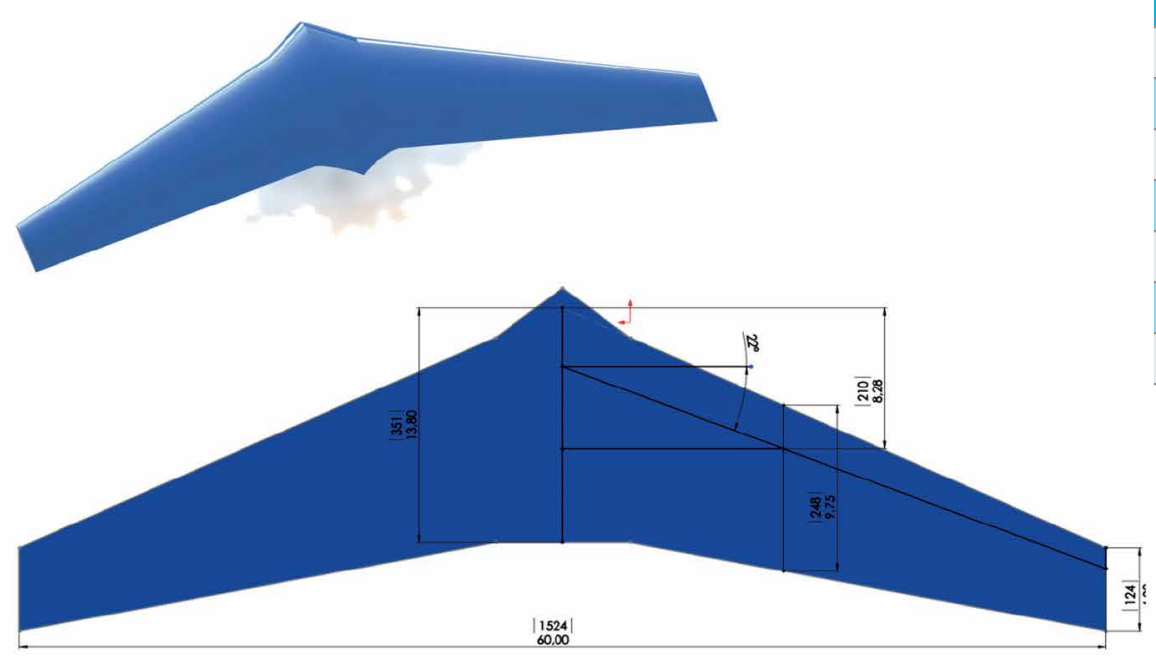

Figura 4. Descripción y dimensionamiento del modelo SU-1. Fuente: elaboración del autor.
Sky, Up/SU-1

\begin{tabular}{|l|l|}
\hline Descripción & \multicolumn{1}{|c|}{$\mathrm{SU}-2$} \\
AR & 6,5 \\
\hline Envergadura & $1,54 \mathrm{mts}$ \\
\hline Área & $0.36 \mathrm{~m} 2$ \\
\hline TR & 0,26 \\
\hline MAC & $25.4 \mathrm{~cm}$ \\
\hline$C_{\text {raiz }}$ & $37 \mathrm{~cm}$ \\
\hline$C_{\text {punta }}$ & $9,4 \mathrm{~cm}$ \\
\hline $\boldsymbol{\Lambda}$ & $24^{\circ}$ \\
\hline
\end{tabular}

$\underset{\text { sacta }}{\text { Sky }}$ Up/SU-1

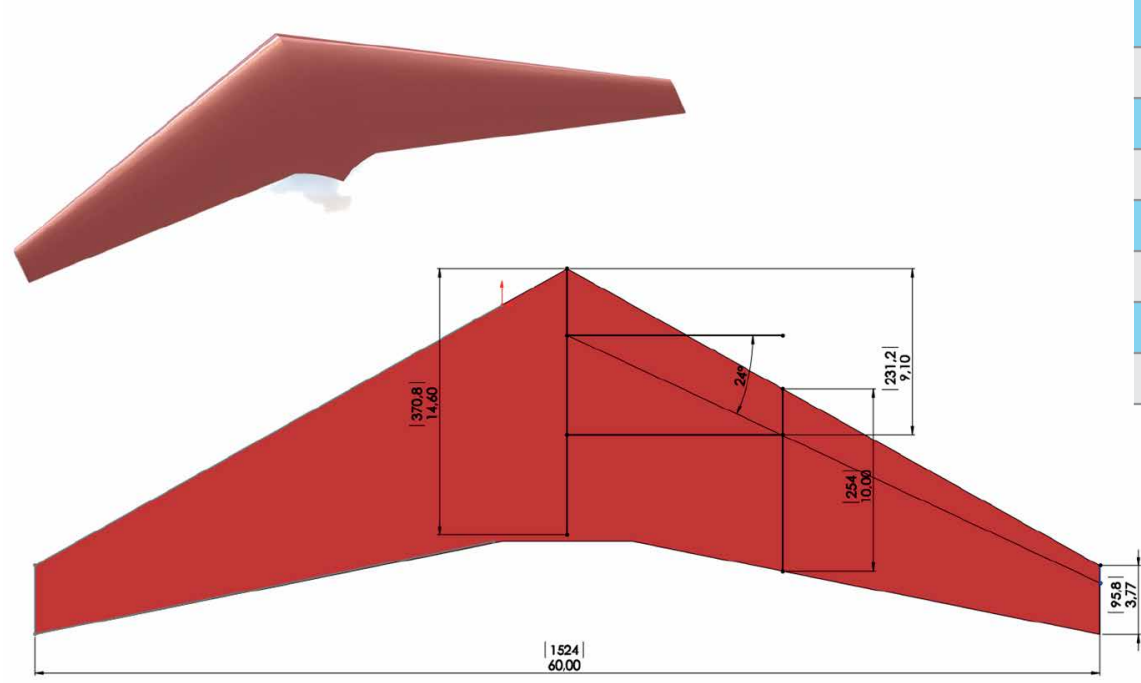

Sky, Up/SU-2

\begin{tabular}{|l|l|}
\hline Descripción & \multicolumn{1}{|c|}{$\mathrm{SU}-2$} \\
\hline AR & 6,5 \\
\hline Envergadura & $1,54 \mathrm{mts}$ \\
\hline Área & $0.36 \mathrm{~m} 2$ \\
\hline TR & 0,36 \\
\hline MAC & $24.7 \mathrm{~cm}$ \\
\hline$C_{\text {raiz }}$ & $35 \mathrm{~cm}$ \\
\hline$C_{\text {punta }}$ & $12,7 \mathrm{~cm}$ \\
\hline$\Lambda$ & $21,62^{\circ}$ \\
\hline
\end{tabular}

Figura 5. Descripción y dimensionamiento del modelo SU-2. Fuente: elaboración del autor. 
$\mathrm{CL}$

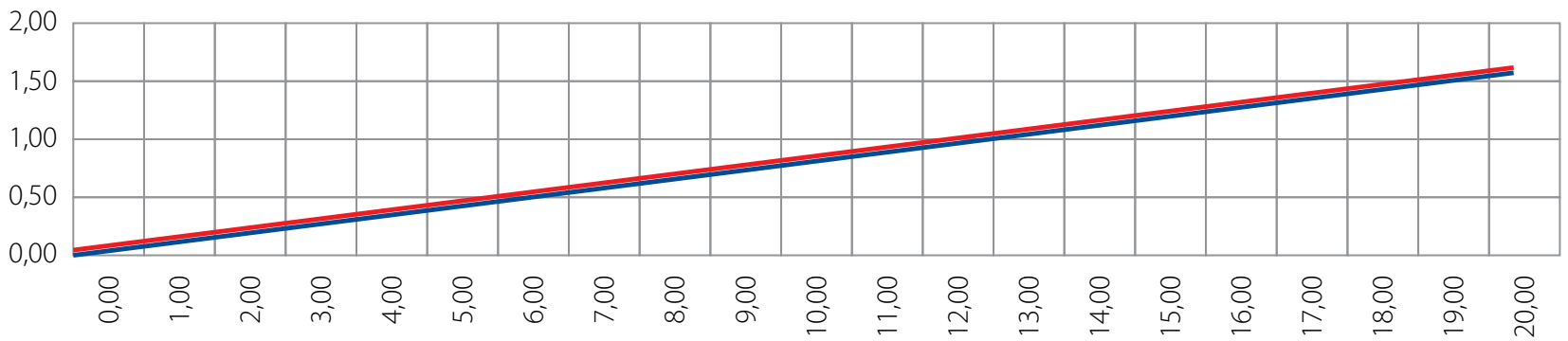

- SU-1 - SU-2

Figura 6. Gráfica de resultados del Coeficiente de Sustentación para SU-1 y SU-2 en relación al ángulo de ataque. Fuente: elaboración del autor.

L/D

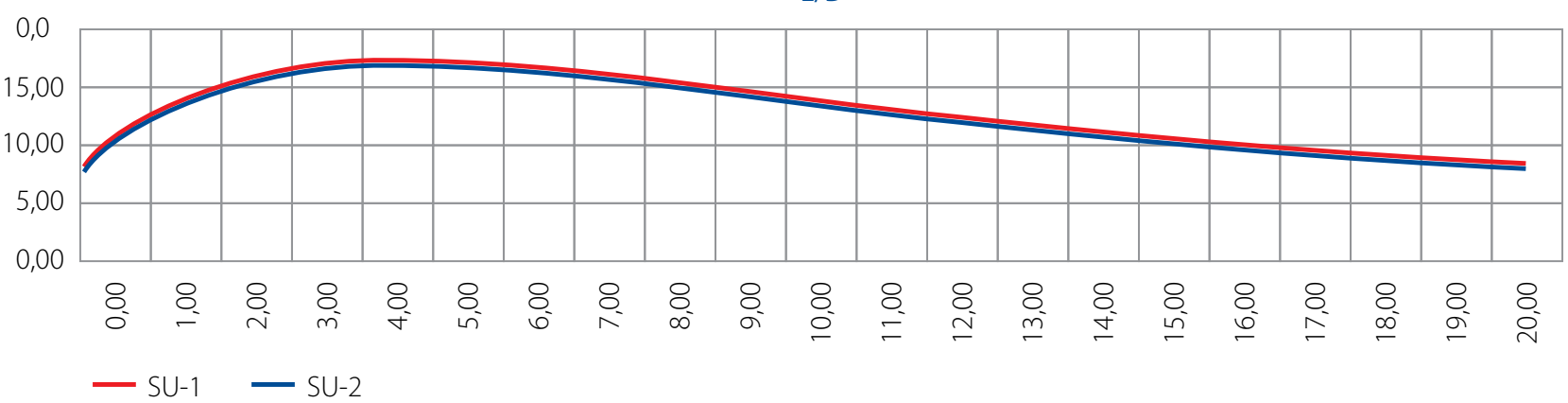

Figura 7. Gráfica de resultados de la relación Sustentación- Arrastre para SU-1 y SU-2. Fuente: elaboración del autor.

\section{Análisis comparativo conceptual variando la forma geométrica de la planta alar \\ El primer análisis comparativo de las geometrías que} se realizó fue basado en el Coeficiente de Sustentación (CL) y la relación Sustentación- Arrastre (L/D) desde la diferencia existente de Razón de Taperado (TR) y el ángulo de aflechamiento $(K)$ que cada modelo tiene (ver Tabla 1), lo que se quiere establecer es la mejor geometría alar para el modelo a desarrollar sin variar el área (S), envergadura (b) ni la razón de aspecto (AR) que ya se determinaron en el diseño preliminar. De esta manera, buscamos cual tiene mejor sustentación a distintos ángulos de ataque, ahora tomando ecuaciones (Raymer, D. P., 2010) para determinar el coeficiente de sustentación a distintos ángulos de ataque se grafica los resultados de ambos modelos (SU-1 \& SU-2).

En la Figura 6, el CL se ve que ambas líneas tienen un comportamiento ascendente de la sustentación a diferentes ángulos de ataque sin ninguna variación entre los modelos analizados, para este caso se establece que variando ángulo de aflechamiento y la razón de taperado la sustentación resultante va ser igual para cualquier modelo. Por consiguiente, se podría utilizar cualquier geometría desde este análisis comparativo, aunque debemos señalar que este análisis inicial no tiene en cuenta el desprendimiento de la capa límite del perfil sobre el extradós de la geometría alar al aumentar el ángulo de ataque como se muestra en la Figura 7 generando una pendiente ascendente lineal, esto se debe a que no se tiene en cuenta la viscosidad del fluido en el momento de calcular el CL para ambos modelos tanto la línea roja que pertenece al análisis del SU-1 y la línea azul del SU-2 esto se debe a que el análisis fue echo asumiendo un fluido no viscoso de lo contrario las líneas de sustentación presentarían gráficamente una pérdida de sustentación a altos ángulos de ataque lo que no es relevante, por ahora, debido a que ambos diseños presenta un idéntico comportamiento en el aumento de sustentación lo que de seguro pasaría igual en la perdida de sustentación.

En los gráficos polar y de Coeficiente de Sustentación (CL) describen la relación sustentación - arrastre con respecto a la variación del ángulo de ataque para los dos modelos, para ambas propuestas encontramos los aspectos más importantes de la curva de L/D es que se haya logrado la misma pendiente e igual valor máximo con los dos análisis desarrollados; lo encontrado es que no im- 
porta la geometría alar, la eficiencia de la aeronave va ser idéntica para el coeficiente CL y la relación L/D.

\section{Estudio comparativo de la geometría alar por número Reynolds en cada cuerda.}

Uno de los parámetros primordiales en el diseño de alas es la selección del perfil el cual se determina dependiendo la configuración física de la aeronave y la misión que debe cumplir, para el diseño aerodinámico de la góndola se seleccionaron dos perfiles, uno para la raíz de la alas, y otro para la punta esta variación de perfiles, a lo largo de la envergadura se denomina entorchamiento aerodinámico (Anderson, J., D., Jr., 2001), el cual tiene como objetivo hacer que la aeronave entre en pérdida de sustentación, primero en la raíz que las punta de las alas con el fin de no entrar en pérdida la aeronave en bajas velocidades y altos ángulos de ataque; el perfil para la raíz del ala es el MH60 y para la punta HS522, los cuales fueron estudiados, con características que cumplen: un coeficiente de momento cercano a cero, curvatura reversa moderada, espesor de 10\% aproximadamente de la cuerda localizado entre el 20 y el $25 \%$, Coeficiente de Arrastre (CD) bajo, pérdida moderada y posibilidad de operar en rangos de número Reynolds por debajo de 150000 como se muestran en las Figuras 9 y 10 las característica de cada perfil a través de una simulación en 2D en el Programa

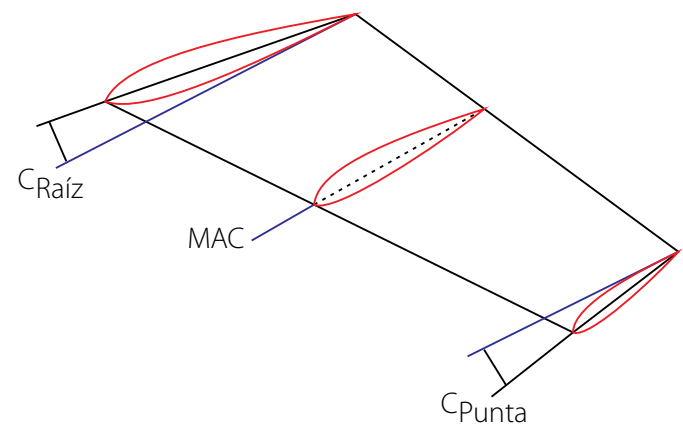

de código libre XFRL5, dichas características son necesarias para alas volantes. Ahora, como el modelo SU1 y SU2 tienen los mismos perfiles es aquí donde se encuentra la más relevante diferencia debido a que la Cuerda en la Raíz $\left(C_{\text {raiz }}\right)$, Cuerda Media Aerodinámica (MAC) y Cuerda en la Punta $\left(C_{\text {punta }}\right)$ varían en su longitud (ver Tabla 2$)$, dicha diferencia nos hace calcular el número Reynolds (RE) para cada curda mediante la siguiente ecuación:

$$
\boldsymbol{R}=V \times C \times 778
$$

Para determinar los valores de RE que se presentaran en la plataforma se usa los valores de las cuerdas preliminares del ala tanto para el SU-1 como para el SU-2 velocidades mínimas o de pérdida $\left(V_{s}\right)$, ya que el valor de $R E$ se encuentra en función de la longitud y de la velocidad.

Teniendo el número Reynolds para cada cuerda de los modelos SU-1 y SU-2 se hizo un estudio en 2D de los perfiles, analizando su comportamiento aerodinámico en el programa XFRL5, para las cuerdas en que el perfil es MH60 tanto el MAC como Craíz; en las dos configuraciones de geometría alar el comportamiento aerodinámico es similar en coeficiente de sustentación, coeficiente de arrastre y coeficiente de momento.

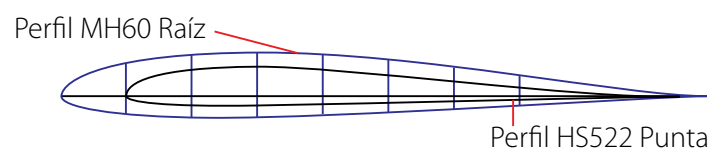

Entorchamiento Aerodinámico

Figura 8. Caracterización del ala. Fuente: elaboración del autor.

Tabla 2. Reynolds para cada cuerda.

\begin{tabular}{ccccccc}
\hline CUERDA & \multicolumn{2}{c}{ SU-1 } & RE (SU-1) & SU-2 & RE (SU-2) \\
\hline MAC & $25.4 \mathrm{~cm}$ & 10 pulg & 186720 & $24.8 \mathrm{~cm}$ & 9.6 pulg & 179251 \\
& & & & & & \\
\hline$C_{\text {raiz }}$ & $37 \mathrm{~cm}$ & 14.5 pulg & 270744 & $35 \mathrm{~cm}$ & 13.7 pulg & 255806 \\
\hline$C_{\text {punta }}$ & $9,5 \mathrm{~cm}$ & 3.7 pulg & 67589 & $12,4 \mathrm{~cm}$ & 4,9 pulg & 91492 \\
\hline
\end{tabular}

Fuente: elaboración del autor. 


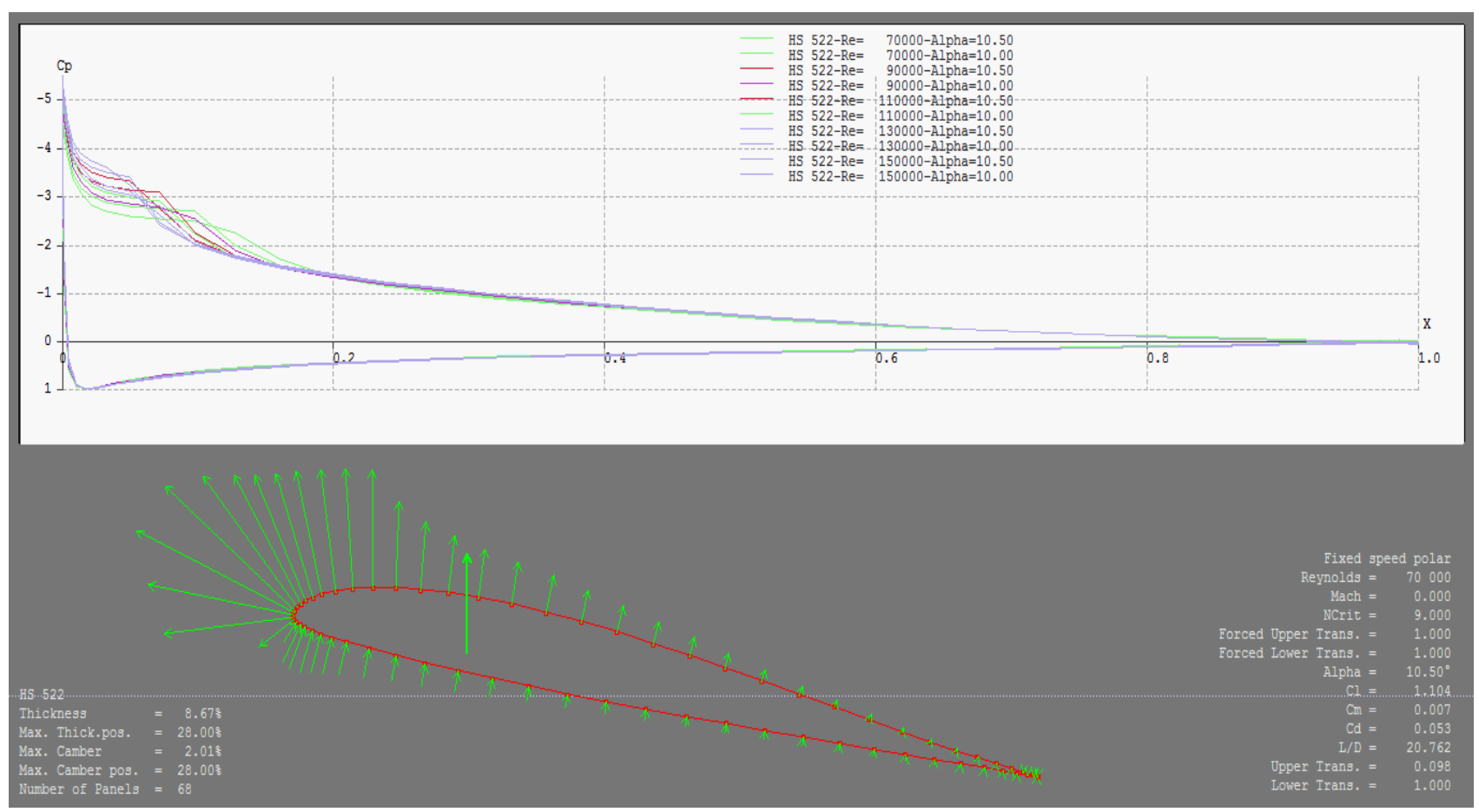

Figura 9. Perfil MH60 Análisis en 2D en XFRL5. Fuente: elaboración del autor.

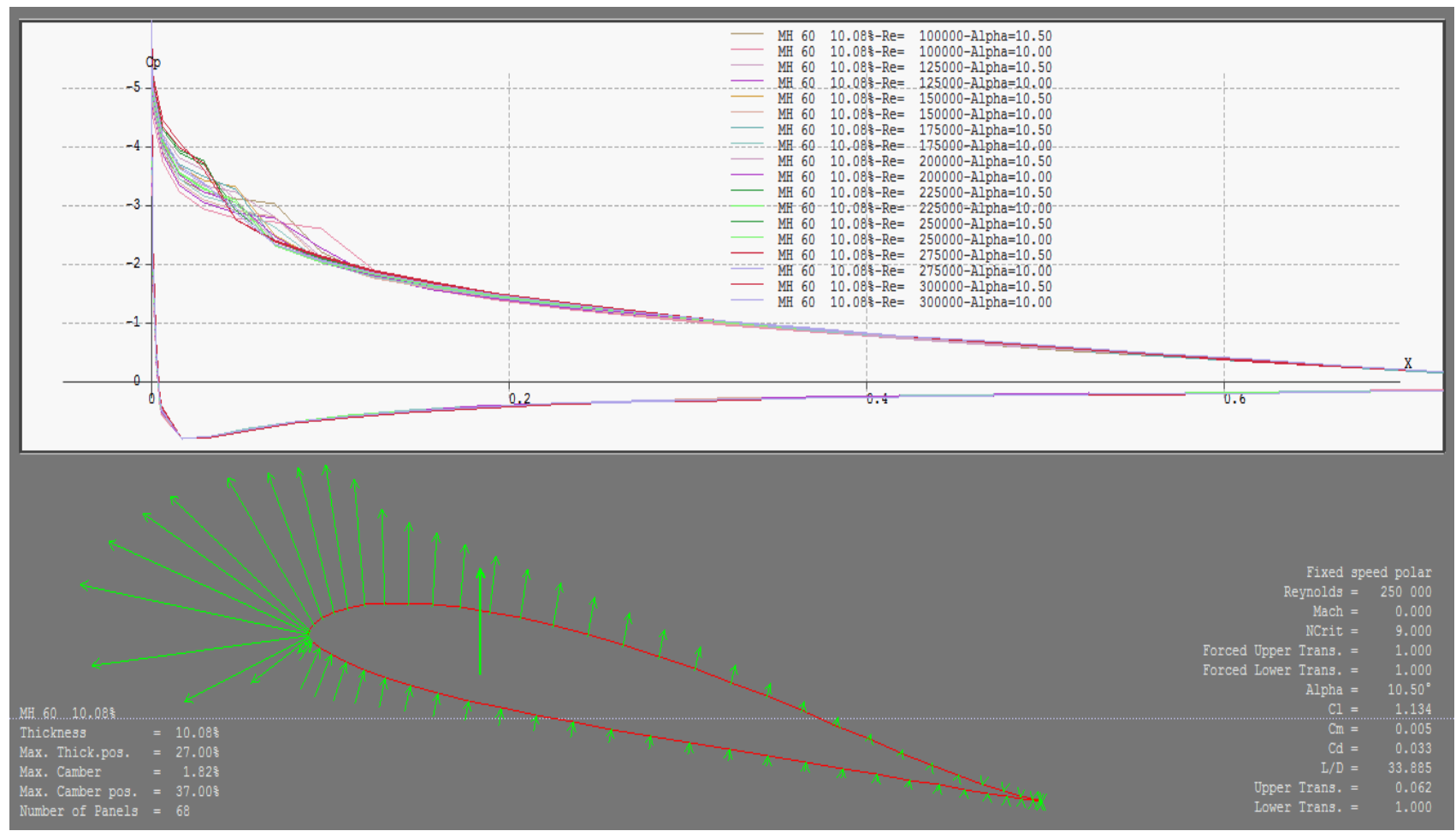

Figura 10. Perfil HS522 Análisis en 2D en XFRL5. Fuente: elaboración del autor. 

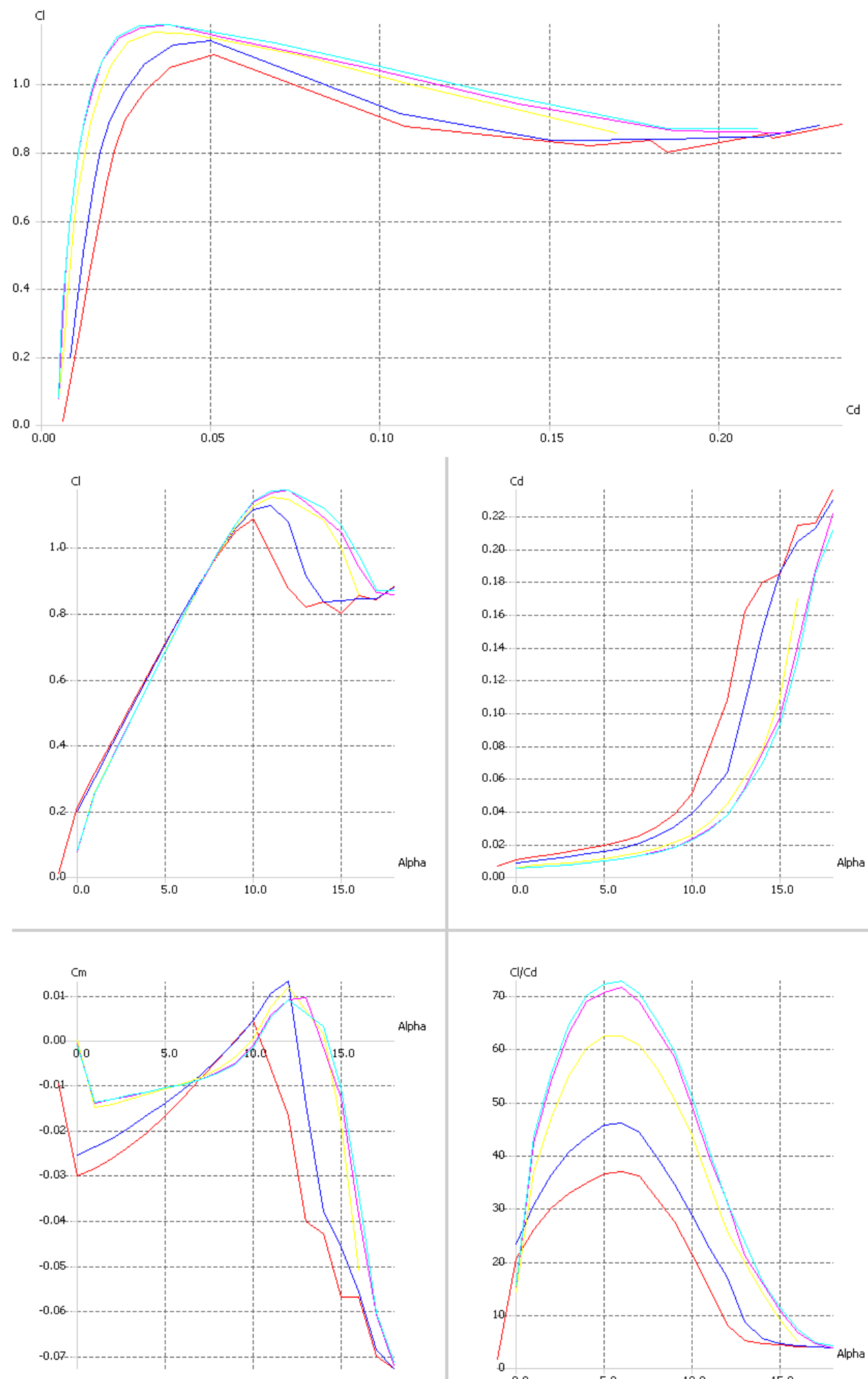

MH $6010.08 \%$

T2_ReO.183_Mo.00 N9.0

HS 522

$$
\begin{aligned}
& \text { — T2_Re0.260_MO.00_N9.0 } \\
& \text { — T2 Re0.067 MO.00 N9.0 } \\
& \text { T2_Re0.275_MO.00_N9.0 T2_ReO.094_MO.00_N9.0 }
\end{aligned}
$$

Figura 11. Curvas de los perfiles MH60 y HS522 a los diferentes números Reynolds. Fuente: elaboración del autor. 
CIENCIA Y PODER AÉREO | Revista Científica de la Escuela de Postgrados de la Fuerza Aérea Colombiana | Vol. 10 | Enero - Diciembre de 2015

\section{Conclusiones}

Según el estudio realizado hasta el momento la configuración de la geometría alar del modelo SU-2 presenta mejor rendimiento para la góndola recuperable y reutilizable al tener mejor coeficiente de sustentación en la punta del ala por el simple hecho de tener más longitud de cuerda en relación al modelo SU-1.

Aunque con este trabajo no es suficiente para tomar una decisión porque hasta el momento se ha analizado la geometría que tiene cada modelo y los perfiles aerodinámicos seleccionados por separado es necesario hacer un estudio en conjunto y en 3D mediante simulaciones en CFD, este análisis computacional se podría hacer en un software especializado en fluidos, hacerlo en XFRL5 que también cuenta con la opción de 3D y por cálculos para cada modelo haciendo una comparación del comportamiento aerodinámico en vuelo, también desarrollar prototipos para hacer algunas pruebas físicas de planeo.

Adicionalmente, se puede analizar ambos modelos con modificaciones aerodinámicas que puedan aumentar la eficiencia de las alas del SkyUp como el de instalarle por ejemplo unos "Winglets" en las puntas de las alas que ayudaría a incrementar el rendimiento del prototipo debido a que su función es reducir los vórtices que se generarían en la punta del ala si se estudia cada modelo tanto el SU1 y SU-2, haciendo esto ayudará a una elección óptima para las exigencias de la misión primordial de recuperación y reutilización del sistema de radio sondeo.

Por otro lado, el nuevo estudio nos ayudaría a confirmar si los perfiles que se han determinado hasta el momento son los indicados para nuestra aeronave, debido a que es posible que los resultados que se logren nos lleven a una reforma de perfiles lo que sería muy útil en la optimización del diseño preliminar a pesar del tiempo que nos tomaría elegir un nuevo perfil o también tendríamos la posibilidad de afianzar los estudios realizados a los perfiles actualmente escogidos y poder lograr el tan anhelado primer lanzamiento.

\section{Referencias}

Anderson, J., D., Jr. (2001). En Fundamentals of aerodynamics. 3ed. Estados Unidos: Mc-Graw Hill.

Hiscocks, R. D., (1995). Design of Light Aircraft. Canada, Vancouver.

Hollman, M.(2002). Modern Aircraft Design, Volumen I, $6^{\text {th }}$ California: Ed. Monterey.

Raymer, D. P., (2010). Aircraft Design a Conceptual Approach. $4^{\text {th }}$ Ed, Washington DC: AIAAA Education Series.
Roskam, J., (1985). Air Plane Design Parte I. Rt4, Ottawa, Kansas.

Stinton, D. (1998). The Anatomy of the Airplane, Second Edition. UK: Leicestershire,

Tecno Esufa. (diciembre, 2013). Revista de Tecnología Aeronáutica, 20. Bogotá D.C.: Escuela de Suboficiales de la Fuerza Aérea Colombiana. 